\title{
Plasma Homocysteine Is a Predictive Factor for Accelerated Renal Function Decline and Chronic Kidney Disease in a Community-Dwelling Population
}

\author{
Wenkai Xiao Ping Ye Fan Wang Ruihua Cao Yongyi Bai Xiaona Wang \\ Department of Geriatric Cardiology, Second Medical Center, Chinese PLA General Hospital, Beijing, China
}

\section{Keywords \\ Homocysteine - Estimated glomerular filtration rate . \\ Chronic kidney disease - Prospective study . General population}

\begin{abstract}
Background: Whether elevated plasma total homocysteine (tHcy) is a risk factor for the progression of kidney disease in general population has not been well established. The purpose of this study was to investigate the prognostic properties of plasma tHcy for renal function decrement and early chronic kidney disease (CKD) in community-dwelling populations with normal renal function at baseline. Methods: A total of 1,426 participants were enrolled and followed for a median of 4.8 years (interquartile range, $4.5-5.2$ ), and estimated glomerular filtration rate (eGFR) was evaluated. One main outcome was the rapid eGFR decline defined as a decline in eGFR of $>3 \mathrm{~mL} / \mathrm{min}$ per $1.73 \mathrm{~m}^{2}$ per year; the other was the new incidence of CKD. Results: At the end of followup, the incidence of rapid eGFR decline and new-onset CKD was 20.7 and $5.6 \%$, respectively. In multivariate linear regression analysis, age, central pulse pressure, fasting blood glucose, and concentration of tHcy were independent determinants of the change in eGFR. There was a graded association between thcy quartiles and eGFR decline. Compared with
\end{abstract}

karger@karger.com www.karger.com/kbr

Karger"

GOPEN ACCESS
(C) 2021 The Author(s)

Published by S. Karger AG, Basel

This is an Open Access article licensed under the Creative Commons Attribution-NonCommercial-4.0 International License (CC BY-NC) (http://www.karger.com/Services/OpenAccessLicense), applicable to the online version of the article only. Usage and distribution for commercial purposes requires written permission. participants with the lowest quartile of thcy levels, those with the highest quartile had significantly increased risk for rapid eGFR decline (adjusted odds ratio $[\mathrm{aOR}]=1.81 ; 95 \%$ confidence interval [Cl]: 1.25-2.94) and new onset of CKD (adjusted hazard ratio $=4.29 ; 95 \% \mathrm{Cl}: 1.42-12.99$ ) after adjusting for various confounders. Similarly, significant associations were also found when baseline tHcy was classified as hyperhomocysteinemia ( $>15 \mu \mathrm{mol} / \mathrm{L}$ ) versus normal tHcy level $(\leq 15 \mu \mathrm{mol} / \mathrm{L})$. However, there was only association between the change in $\mathrm{tHcy}$ levels and new occurrence of CKD but not with rapid eGFR decline ( $\mathrm{aOR}=0.99, p=0.613$ ). Conclusions: In this prospective cohort of individuals from community-based population, elevated plasma tHcy emerged as an independent predictor of renal function decline and incident CKD, which might support selection of at-risk individuals.

(C) 2021 The Author(s).

Published by S. Karger AG, Basel

\section{Introduction}

Chronic kidney disease (CKD) is a growing public health challenge worldwide. The prevalence of CKD is high in developing countries, and China has the largest number of CKD patients in the world, reaching about 147 million [1]. Considering the high prevalence of morbid-
Correspondence to:

Ping Ye, yeping301@sina.com 
ity and mortality associated with CKD, it is clinically important to identify risk factors responsible for accelerated renal function decline and apply the necessary preventive measures. It has been well documented that advanced age, diabetes mellitus, hypertension, proteinuria, and smoking are associated with subsequent reduction in estimated glomerular filtration rate (eGFR) [2-3]. Despite prevention and treatment of these established factors, the number of patients with CKD is increasing [4], suggesting that traditional factors alone are inadequate to explain CKD risks and improve risk stratification, and other factors are involved in renal function deterioration, and they must be evaluated.

Homocysteine is a sulfur-containing amino acid that functions as an intermediate in methionine metabolism [5]. Even a moderate elevation in homocysteine levels is widely recognized as a risk factor for cardiovascular and cerebrovascular disease [6-8]. Previous cross-sectional studies have demonstrated that the prevalence of hyperhomocysteinemia (HHcy) in CKD patients was significantly higher than that in healthy people [9], and the plasma homocysteine level was negatively associated with eGFR in general population [10-11]. Recently, similar pathological changes were observed in glomerular injury and total homocysteine (tHcy)-induced vascular damages, such as endothelial dysfunction, cell proliferation, increased oxidative stress, and decreased production of adenosine [12-13]. On the basis of these findings, HHcy may be a pathogenic factor resulting in renal injury and accelerated deterioration of renal function, rather than only resulting as a functional consequence of reduced renal function. Yet, long-term renal function decline with respect to elevated tHcy levels has not been assessed properly, and the results were controversial [14-15]. China is a country without folic acid fortification, and HHcy is more common than other countries [16]. This necessitates further investigation into HHcy and renal function change among the Chinese population.

The purpose of this study is to evaluate whether elevated tHcy is a substantial determinant of renal function deterioration and can independently predict the risk of CKD. Thus, in the current study, we aimed to prospectively investigate the associations of elevated tHcy levels with the risk of renal function aggravation in an apparently healthy Chinese community-dwelling cohort with normal renal function (eGFR $\geq 60 \mathrm{~mL} / \mathrm{min}$ per $1.73 \mathrm{~m}^{2}$ ) at baseline.

\section{Methods}

\section{Study Population}

This current study reports the results of a community-based cohort study of people living in the Pingguoyuan communities of Shijingshan district in Beijing, China. In brief, between September 2007 and January 2009, a total of 1,631 participants without clinically apparent cardiovascular disease (CVD) were initially recruited for cross-sectional analysis of CVD risk factors. In this enrolled population, we further excluded 30 participants, who already had CKD at baseline. After the initial assessment, enrolled subjects were contacted every 2 years for follow-up, and the last follow-up visits were conducted through September 30, 2013, and the median follow-up was 4.8 (interquartile range 4.5-5.2) years. During the period between the initiation of the study and the follow-up, 52 subjects died (cause of death consists of 23 cardio-cerebrovascular diseases, 13 pulmonary diseases, 9 malignant tumor, 1 hematopathy, and 6 were unknown), and 175 participants were lost to follow-up and were excluded from the analysis. No differences other than baseline risk factors were noted in those who completed baseline and follow-up assessments. Complete follow-up data were obtained from 1,426 participants (return rate, 89.1\%).

\section{Data Collection}

Clinical Data

Baseline data for these analyses were collected using self-reported standardized questionnaires that included demographics, life-style information, medical history, and medication use. The medication use was validated by examination of pills and prescriptions brought to the clinic for that purpose. Anthropometrics were evaluated by trained medical doctors, and the BMI was derived from height and weight measured with participants wearing light clothing and no shoes. Blood pressures were measured manually using a mercury sphygmomanometer (Yuwell Medical Equipment \& Supply Co., Ltd.) and a standard-sized cuff by trained personnel. Two or more measurements separated by at least 5 min were taken from the right arm after 5 min of rest in a sitting position, and the average of at least 2 readings was used for further analysis. Then, central arterial BP and wave reflection were assessed in a sitting position after $\geq 5$ min of rest using the SphygmoCor pulse wave analysis system (AtCor Medical) via the right radial artery at the same sites; details of the measurement have been described previously in a published document [17].

\section{Biomarker Assays}

Baseline blood samples were collected from participants between 8:00 a.m. and 10:00 a.m. after a fast of at least $12 \mathrm{~h}$ at enrollment. Follow-up measures were performed on blood samples collected 4-5.5 years later, and serum or plasma samples were separated within $30 \mathrm{~min}$ of collection. Serum creatinine was measured using an enzymatic method by the Roche enzymatic assays (Roche Diagnostics $\mathrm{GmbH}$ ) on a Hitachi 7,600 automatic analyzer (Hitachi). Plasma homocysteine was determined by high-performance liquid chromatography using fluorescence detection according to the modified method of Araki et al. [18]. HHcy was defined as plasma tHcy concentration $>15 \mu \mathrm{mol} / \mathrm{L}$ according to the recent clinical literature [19-20]. Concentrations of glucose (fasting and $120 \mathrm{~min}$ after an oral glucose load), total cholesterol, triglycerides, high-density lipoprotein cholesterol (HDL-C), low-density lipoprotein cholesterol (LDL-C), and uric acid levels were measured 
Table 1. Characteristics of participants according to the quartiles of homocysteine at baseline

\begin{tabular}{|c|c|c|c|c|c|}
\hline \multirow[t]{2}{*}{ Variables } & \multicolumn{4}{|c|}{ Homocysteine, $\mu \mathrm{mol} / \mathrm{L}$} & \multirow[t]{2}{*}{$p$ value } \\
\hline & quartile $1,<13.6$ & quartile $2,13.6-16.7$ & quartile $3,16.8-20.8$ & quartile $4, \geq 20.9$ & \\
\hline Age, years & $53.17 \pm 10.09$ & $58.12 \pm 9.87$ & $62.18 \pm 10.02$ & $63.23 \pm 11.41$ & $<0.001$ \\
\hline Male, $n(\%)$ & $71(20.06)$ & $112(31.11)$ & 149 (41.97) & $275(77.03)$ & $<0.001$ \\
\hline $\mathrm{BMI}, \mathrm{kg} / \mathrm{m}^{2}$ & $25.26 \pm 3.30$ & $25.14 \pm 3.28$ & $25.45 \pm 3.37$ & $26.04 \pm 3.34$ & 0.003 \\
\hline Hypertension, $n(\%)$ & $123(34.75)$ & $162(45.00)$ & $176(49.58)$ & $192(53.78)$ & $<0.001$ \\
\hline Diabetes mellitus, $n(\%)$ & $58(16.38)$ & $73(20.28)$ & $77(21.69)$ & $56(15.69)$ & 0.108 \\
\hline Systolic BP, mm Hg & $124.82 \pm 16.84$ & $127.75 \pm 16.29$ & $129.28 \pm 16.73$ & $134.73 \pm 18.87$ & $<0.001$ \\
\hline Diastolic BP, mm Hg & $75.61 \pm 9.87$ & $77.25 \pm 9.81$ & $76.79 \pm 10.23$ & $78.71 \pm 10.60$ & 0.002 \\
\hline Total cholesterol, mmol/L & $5.00 \pm 0.95$ & $5.16 \pm 0.93$ & $5.00 \pm 0.97$ & $4.95 \pm 0.87$ & 0.033 \\
\hline Triglycerides, mmol/L & $1.70 \pm 1.22$ & $1.85 \pm 1.16$ & $1.73 \pm 1.05$ & $1.86 \pm 1.24$ & 0.187 \\
\hline Uric acid, $\mu \mathrm{mol} / \mathrm{L}$ & $261.96 \pm 67.46$ & $297.44 \pm 73.75$ & $296.02 \pm 69.79$ & $319.27 \pm 79.66$ & $<0.001$ \\
\hline Serum creatinine, $\mu \mathrm{mol} / \mathrm{L}$ & $61.36 \pm 13.10$ & $64.37 \pm 15.78$ & $67.18 \pm 16.20$ & $75.87 \pm 16.98$ & $<0.001$ \\
\hline $\mathrm{EGFR}, \mathrm{mL} / \mathrm{min} / 1.73 \mathrm{~m}^{2}$ & $103.07 \pm 13.13$ & $97.49 \pm 13.48$ & $94.16 \pm 14.43$ & $90.42 \pm 18.43$ & $<0.001$ \\
\hline NT-proBNP, pg/mL & $34.6(16.9,64.5)$ & $33.1(17.9,63.6)$ & $46.7(19.1,86.1)$ & $37.6(19.1,85.4)$ & 0.021 \\
\hline Hs-CRP, mg/L & $2.40(1.60,3.40)$ & $2.10(1.30,3.40)$ & $2.10(1.30,3.30)$ & $2.25(1.30,3.40)$ & 0.505 \\
\hline Central SBP, mm Hg & $115.92 \pm 16.77$ & $118.76 \pm 18.04$ & $119.71 \pm 16.81$ & $122.91 \pm 17.51$ & $<0.001$ \\
\hline Central DBP, mm Hg & $76.61 \pm 9.87$ & $77.25 \pm 9.82$ & $76.79 \pm 10.23$ & $78.71 \pm 10.59$ & 0.002 \\
\hline Central PP, mm Hg & $49.21 \pm 12.82$ & $50.50 \pm 13.19$ & $52.49 \pm 13.66$ & $56.02 \pm 15.67$ & $<0.001$ \\
\hline \multicolumn{6}{|l|}{ Medication use } \\
\hline Antihypertensives, $n(\%)$ & $78(22.03)$ & $106(29.44)$ & $129(36.34)$ & $147(41.18)$ & $<0.001$ \\
\hline Antidiabetics, $n(\%)$ & $31(8.76)$ & $43(11.94)$ & $42(11.83)$ & $38(10.64)$ & 0.489 \\
\hline
\end{tabular}

Values are reported as $n(\%)$, mean $\pm \mathrm{SD}$, or median (interquartile range). BP, blood pressure; HDL, high-density lipoprotein; LDL, low-density lipoprotein; FBG, fasting blood glucose; PBG, postprandial blood glucose; eGFR, estimated glomerular filtration rate; NTproBNP, N-terminal pro-type B natriuretic peptide; Hs-CRP, high-sensitivity C-reactive protein; SBP, systolic blood pressure; DBP, diastolic blood pressure; PP, pulse pressure.

by routine laboratory analysis on a Roche autoanalyzer. N-terminal pro-B-type natriuretic peptide (NT-proBNP) concentrations were determined with an electrochemiluminescence immunoassay (Roche Diagnostics GmbH) using a Roche analyzer. Levels of high-sensitivity C-reactive protein (Hs-CRP) were determined by an immunoturbidimetric assay (Siemens Healthcare Diagnostics, Elkhart, IN, USA) using a Dimension RxL Max analyzer (Siemens Healthcare Diagnostics). All testing was performed by well-trained personnel blinded to clinical data in the Department of Biochemistry of Chinese PLA General Hospital.

\section{Definition of Variables}

The eGFR was calculated using the Chronic Kidney Disease Epidemiology Collaboration (CKD-EPI) equation, and details of the computing equation have been published elsewhere [21]. Changes in eGFR over time were calculated as the difference between the eGFR at follow-up and baseline, and positive values in- dicate a decline of eGFR from baseline to the end of follow-up. One main renal outcome of interest was the rapid eGFR decline, defined as a decline in eGFR of $>3 \mathrm{~mL} / \mathrm{min}$ per $1.73 \mathrm{~m}^{2}$ per year; the other was the new incidence of CKD, defined as a decrease in eGFR of $20 \%$ or more and to a level of below $60 \mathrm{~mL} / \mathrm{min}$ per $1.73 \mathrm{~m}^{2}$ at the end of follow-up.

\section{Statistical Analysis}

Baseline characteristics were stratified by tHcy quartiles, and continuous variables are reported as mean \pm standard deviation (SD) or median (interquartile range) and categorical variables as count and percentages; $\mathrm{tHcy}$ level and other biomarkers were normalized by natural logarithm transformation, as necessary. Differences in the clinical characteristics across tHcy quartiles were analyzed using the 1-way analysis of variance for continuous measures, Cuzik's nonparametric trend test for nonnormally distributed variables, and $\chi^{2}$ tests were performed for categorical 
Table 2. Multivariate linear analysis between the change in eGFR and various baseline parameters

\begin{tabular}{lllr}
\hline Parameter & $\begin{array}{l}\text { Standardized } \\
\beta\end{array}$ & $t$ & $p$ value \\
& $\beta$ & & \\
\hline Age (years) & 0.145 & 4.590 & $<0.001$ \\
Central PP (mm Hg) & 0.130 & 3.971 & $<0.001$ \\
Fasting blood glucose (mmol/L) & 0.086 & 3.130 & 0.002 \\
Ln tHcy ( $\mu \mathrm{mol} / \mathrm{L})$ & 0.081 & 2.828 & 0.005 \\
\hline
\end{tabular}

$\beta$ and $p$ values are shown only when $p<0.05$. $\beta$, regression coefficient; PP, pulse pressure; Ln, natural logarithm-transformed; tHcy, total homocysteine.

variables. First, multivariate linear regression models were used to evaluate the independent determinants for change in eGFR and changes in eGFR as a continuous variable.

In addition, to better understand the effects of baseline and the change in tHcy on the occurrence of rapid eGFR decline, logistic regression models were used. Odds ratios (ORs) and 95\% confidence intervals (CIs) were computed in 3 directions: the change in tHcy between baseline and end of follow-up and the HHcy category compared with the normal category and across quartiles of baseline tHcy. We conducted 3 types of models for each analysis: an unadjusted model (model 1); a model adjusted for age, sex, the presence of hypertension or diabetes mellitus, smoking, BMI, brachial mean arterial pressure, fasting blood glucose, HDL-C, LDLC, uric acid, eGFR at baseline, and antihypertensive or hypoglycemic medications use (model 2); and model 3 was adjusted for model 2 plus central pulse pressure, level of NT-proBNP, and Hs-CRP.

The cumulative incidence of CKD for each category of tHcy was evaluated using the Kaplan-Meier method and compared with the log-rank test. Furthermore, multivariable Cox proportional hazards regression models were used to determine associations of the above 3 conditions with incident CKD. Cox regression models were adjusted for the relevant variables as described in the logistic regression models. The adjusted hazard ratios (HRs) are reported with 95\% CIs, and values of $p<0.05$ (2-tailed) were considered statistically significant. All data were analyzed using the SPSS statistical package software (version 25.0; SPSS Inc). A 2-sided value of $p<0.05$ was considered statistically significant.

\section{Results}

\section{Participant Characteristics}

Altogether, we included 1,426 participants at enrollment, and the mean $( \pm \mathrm{SD})$ age of participants was $61.3 \pm$ 11.3 years and $57.4 \%$ were women. The median of plasma tHcy was $16.7 \mu \mathrm{mol} / \mathrm{L}$, and mean of eGFR was $96.3 \mathrm{~mL} /$ min per $1.73 \mathrm{~m}^{2}$ at baseline. Table 1 shows different characteristics according to the baseline tHcy quartiles. Compared with those with lower tHcy levels, individuals with increased tHcy were older; were more likely to be male and frequently hypertensive; had lower eGFR and HDL$\mathrm{C}$ levels; and had higher systolic blood pressure, pulse pressure, and uric acid levels. Taking of antihypertensive, antidiabetic, and antiplatelet varied across levels of tHcy.

During the observation period, the eGFR decreased by $8.2 \%$, corresponding to a yearly change of $1.60 \mathrm{~mL} / \mathrm{min}$ per $1.73 \mathrm{~m}^{2}$ per year, and 80 participants experienced new-onset CKD. The incidence of rapid eGFR decline and CKD was 20.7 and $5.6 \%$, respectively.

\section{Independent Determinants of the Change in Renal Function}

To determine independent predictors of change in renal function, multivariate linear regression analysis was used and changes in eGFR as a continuous variable. As shown in Table 2, the baseline concentration of tHcy was correlated with the decrease in eGFR during follow-up. Other parameters entered in the model were age, central pulse pressure, and fasting blood glucose.

\section{Rapid Renal Function Decline by Initial and the Change in tHcy}

Table 3 displays the results of multivariate logistic regression for effects of baseline tHcy and the change in tHcy on rapid eGFR decline. After adjusted for potential confounders, HHcy was independently associated with increased risk of rapid decline in eGFR (with a fully adjusted OR of 1.53 [95\% CI: 1.15-2.04]). The similar trends were found when tHcy was assessed as quartiles; compared to the lowest quartile, the multivariable-adjusted ORs (95\% CI) for rapid eGFR decline were 1.81 (1.25-2.94) for the highest quartile and $1.72(1.12-2.51)$ for the third quartile of baseline $\mathrm{tHcy}$. By contrast, the change in tHcy was not associated with rapid eGFR decline either in the univariate or in the multivariable logistic regression analysis.

\section{tHcy Categories and Risk of CKD over Time}

The cumulative incidences of CKD by tHcy categories are shown in Figure 1a, b. As demonstrated by the Kaplan-Meier survival analysis, there was a strong and graded increase in the probability of CKD occurrence across categories.

As shown in Table 4, the risk of CKD occurrence was associated with both baseline tHcy and the change in tHcy. In a Cox proportional hazard model, risk for CKD was significantly higher in the HHcy cohort. This relationship remained substantially unchanged, even after adjustment for other confounding factors, such as eGFR at baseline, blood pressures, NT-proBNP, and Hs-CRP ([Table 4, model 3], with a fully adjusted HR of 2.26 [95\% 
Table 3. Association of baseline and the change in tHcy with rapid renal function decline

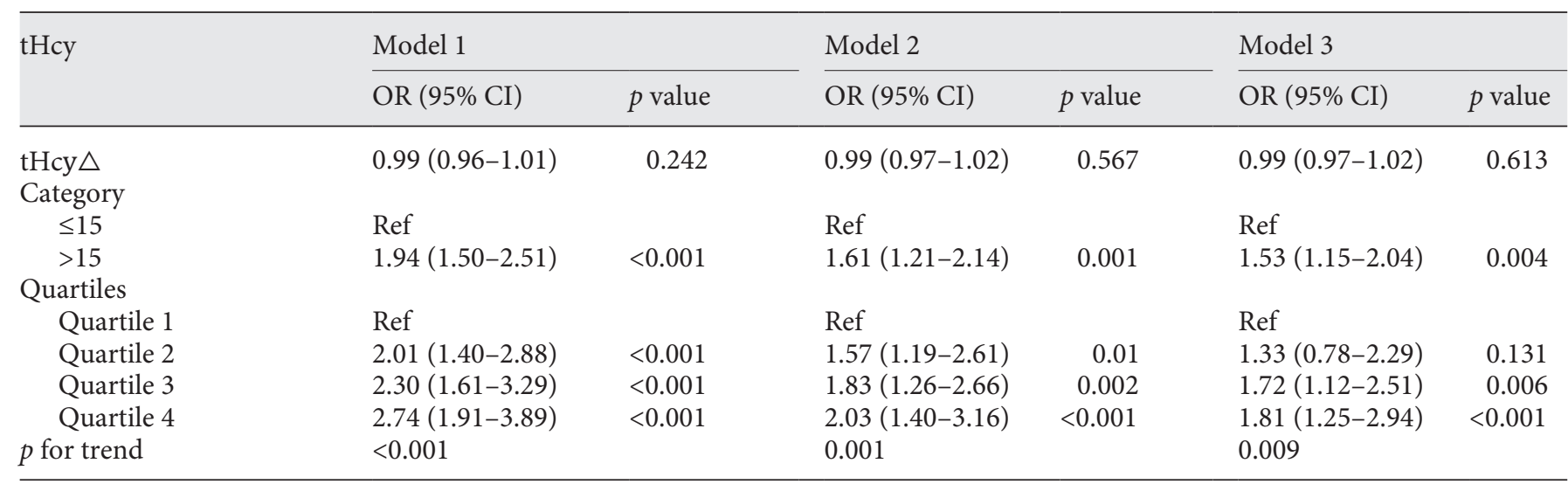

Total homocysteine quartiles: quartile $1<13.6,13.6 \leq$ quartile $2 \leq 16.7,16.8 \leq$ quartile $3 \leq 20.8$, and quartile $4 \geq 20.9$. Models are defined as follows: model 1 is unadjusted; model 2 is adjusted for age, sex, the presence of hypertension or diabetes mellitus, smoking, BMI, brachial mean arterial pressure, fasting blood glucose, HDL cholesterol, LDL cholesterol, uric acid, eGFR at baseline, and antihypertensive or hypoglycemic medications use; model 3 is additionally adjusted for central pulse pressure, $\mathrm{N}$-terminal pro-type B natriuretic peptide, and high-sensitivity C-reactive protein. tHcy $\triangle$, change in total homocysteine (tHcy follow-up - tHcy baseline); OR, odds ratio; CI, confidence interval.

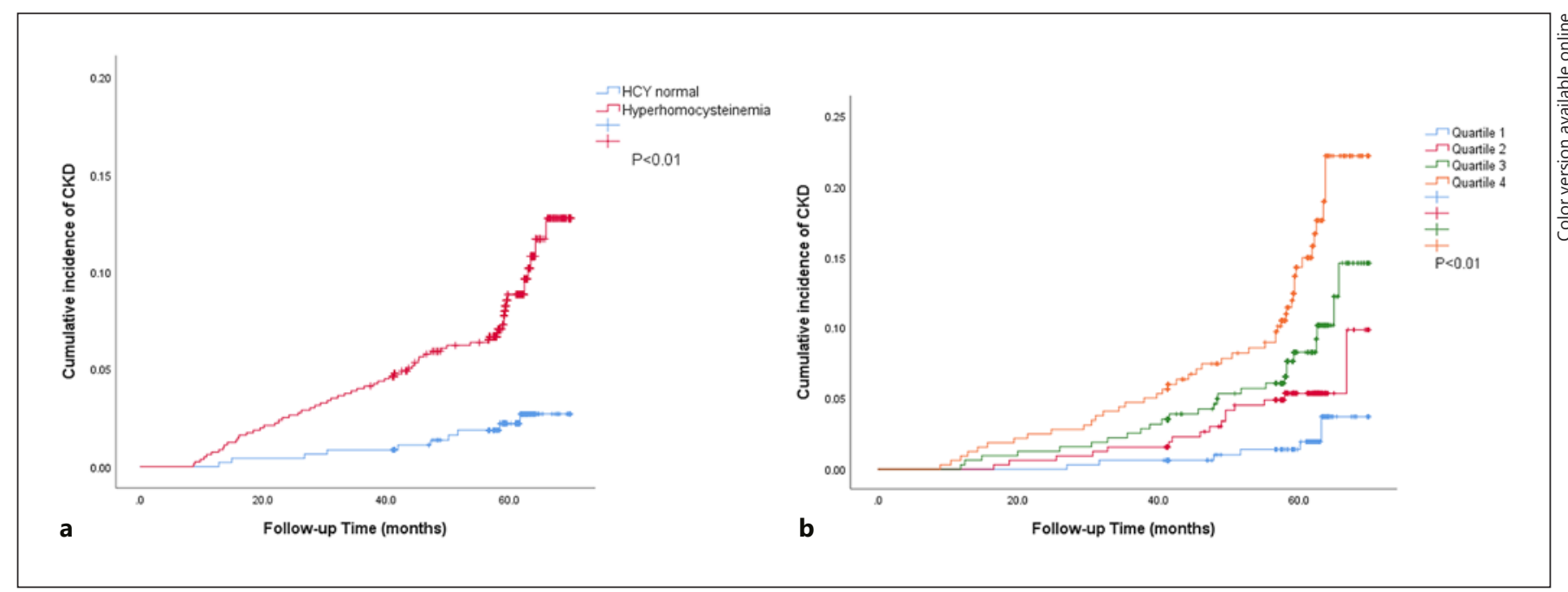

Fig. 1. Kaplan-Meier curves for incident CKD. Kaplan-Meier curves for cumulative incidence of the CKD in the high $(>15$ $\mu \mathrm{mol} / \mathrm{L})$ versus normal $(\leq 15 \mu \mathrm{mol} / \mathrm{L})$ homocysteine category $(\mathbf{a})$ and according to quartiles of plasma homocysteine (b). Groups are indicated by colors; $p<0.01$ for all between-group comparisons by the log-rank test. CKD, chronic kidney disease.
CI: 1.11-4.58]). In analyses that considered quartiles of tHcy, the HR for incident CKD increased across quartiles of plasma tHcy level, reaching 4.29 (95\% CI: 1.42-12.99) for the top quartile compared with the lowest quartile $(p$ for trend 0.017) in fully adjusted models. Unlike rapid eGFR decline, there was a significant association between change in tHcy levels and risk for incident CKD.

\section{Discussion}

The main objective of the present study was to evaluate the usefulness of plasma tHcy for prediction of renal function aggravation and incident CKD in a communitybased study of subjects with initial eGFR $>60 \mathrm{~mL} / \mathrm{min}$ per $1.73 \mathrm{~m}^{2}$ and without clinically apparent CVD at entry. 
Table 4. Multivariate Cox regression analysis of the determinants of new occurrence of CKD

\begin{tabular}{|c|c|c|c|c|c|c|}
\hline \multirow[t]{2}{*}{ tHcy } & \multicolumn{2}{|l|}{ Model 1} & \multicolumn{2}{|l|}{ Model 2} & \multicolumn{2}{|l|}{ Model 3} \\
\hline & HR (95\% CI) & $p$ value & HR (95\% CI) & $p$ value & HR (95\% CI) & $p$ value \\
\hline \multicolumn{7}{|l|}{ Category } \\
\hline$\leq 15$ & Ref & & Ref & & Ref & \\
\hline$>15$ & $3.78(1.94-7.37)$ & $<0.001$ & $2.68(1.33-5.39)$ & 0.006 & $2.26(1.11-4.58)$ & 0.024 \\
\hline \multicolumn{7}{|l|}{ Quartiles } \\
\hline Quartile 2 & $3.90(1.28-11.86)$ & 0.016 & $2.27(1.07-7.06)$ & 0.038 & $1.62(0.83-4.14)$ & 0.119 \\
\hline Quartile 3 & $5.41(1.86-15.73)$ & 0.002 & $3.15(1.34-10.28)$ & 0.01 & $2.43(1.08-6.98)$ & 0.035 \\
\hline Quartile 4 & $8.51(3.02-23.96)$ & $<0.001$ & $5.27(1.77-15.72)$ & $<0.001$ & $4.29(1.42-12.99)$ & 0.01 \\
\hline$p$ for trend & $<0.001$ & & 0.003 & & 0.017 & \\
\hline
\end{tabular}

Multivariab Cox regression models were adjusted for the covariates in models 1,2 , and 3 (the same as described in Table 3 ). tHcy $\triangle$, change in total homocysteine; HR, hazard ratio; CI, confidence interval.

Elevated tHcy indicated a clear association with both accelerated renal function decline and the occurrence of CKD in this prospective cohort study. Also, this risk prediction persisted even after adjustment for traditional cardiovascular risk factors as well as adjustment for baseline eGFR and some biomarkers. Therefore, tHcy may be a useful tool in clinical for prediction of progressive renal disease in the general population.

CKD represents a significant public health problem because of the high prevalence and associated cardiovascular morbidity and mortality. Traditionally, hypertension, diabetes, and proteinuria are well-recognized risk factors for progressive renal function loss [22]. However, these traditional factors are inadequate to predict CKD risk and improve risk stratification for progression of kidney injury [23-24]. Several other pathways may be involved in CKD development including inflammation and endothelial dysfunction [25]. Therefore, early identification of the risk factors for CKD is critical for preventing the development of kidney damage and adverse outcomes. To date, little is known regarding whether elevated tHcy can increase the risk of CKD in Chinese middleaged and elderly population.

The median level of tHcy was $16.7 \mu \mathrm{mol} / \mathrm{L}$, and $62.8 \%$ had HHcy in this study. Concentration of homocysteine varies according to the populations but generally high in men and in old age [16]. HHcy is more common among Chinese population, especially

in the northern urban areas $[16,26]$, mostly due to the local dietary pattern of high intake of refined cereals. Those following the refined cereals pattern were 4 times more likely to have high homocysteine, relative to the fruit and milk dietary pattern group [26]. Another reason is the potential influence of the gene polymorphism of methylenetetrahydrofolate reductase (MTHFR); MTHFR C677T mutation modifies MTHFR function leading to the development of HHcy [27]. Chinese population had a higher prevalence of MTHFR C677T mutation than in Western populations ( $25 \%$ vs. $<10 \%$ ) [28]. Recently, a renal substudy of the China Stroke Primary Prevention Trial (CSPPT) found that the combined use of enalapril and folic acid, compared with enalapril alone, could significantly delay the progression of CKD among patients with mild-to-moderate CKD [29].

To the best of our knowledge, few prospective studies have examined the relationship of elevated $\mathrm{tHcy}$ with the decline of renal function or the risk of CKD in the general population. The Hisayama study [30] investigated 1,477 community-dwelling Japanese individuals aged 40 years or older without CKD and were followed up for 5 years. Notably, after adjustment for baseline creatinine level, elevated tHcy levels were significantly associated with the incidence of CKD in women but not in men. In a historical prospective study on 3,602 subjects in Israel, the research showed that elevated tHcy level was associated with an accelerated decline in renal function in both men and women and was an independent risk factor for the development of CKD in the general population over a median follow-up time of 7.75 years [14]. Also, the independent prediction of HHcy on renal function decline was demonstrated in 2,387 Chinese hypertensive adults without CKD at baseline [31]. However, in 2 prospective 
studies about patients with moderate or severe decline in eGFR $[15,32]$, there were no indications that elevated homocysteine levels play a contributing role for an accelerated kidney disease process. Consistent with previous studies in subjects without CKD, our study revealed a dose-response effect between higher tHcy levels and deterioration of renal function or development of CKD. The effects of HHcy may differ between subjects with normal renal function and those with renal dysfunction; tHcy may be an important risk factor for the early impairment of renal function.

The exact mechanisms underlying the relationship between elevated tHcy and kidney disease progression are not fully understood, and there are several potential explanations for this finding. HHcy-associated renal vascular changes include reduction in vascular density and blood flow and increased smooth muscle cell proliferation contributing to impaired renal function [33-34]. One mechanism is that HHcy reduces the endogenous generation of hydrogen sulfide $\left(\mathrm{H}_{2} \mathrm{~S}\right)$, a well-known vasodilator and major regulator of inflammatory responses [35]. Transcriptional deregulation of genes encoding for $\mathrm{H}_{2} \mathrm{~S}$-producing enzymes was present in CKD, and the levels of $\mathrm{H}_{2} \mathrm{~S}$ were found to be significantly decreased [36]. Low plasma $\mathrm{H}_{2} \mathrm{~S}$ has been linked to decreased glomerular function, while exogenous $\mathrm{H}_{2} \mathrm{~S}$ supplementation can reduce glomerular and tubulointerstitial sclerosis and improve renal perfusion $[12,37]$. Second, nitric oxide (NO)mediated efferent arteriole dilation is an important protective mechanism against glomerular hypertension [38], and elevated homocysteine has been found to reduce NO production and directly trap $\mathrm{NO}$, which over time may cause endothelial dysfunction and damage, activating oxidative stress in the arterial wall [39-40]. Therefore, higher tHcy may inhibit the vasodilation of the renal arteriole, thus enhancing the acceleration of renal injury progression. Third, evidence is accumulating that tHcy may result in a significant increase in reactive oxygen species production, which is paralleled with enhanced sympathetic tone and blood pressure [41]. Recently, a large retrospective study [42] demonstrated that HHcy and hypertension may act synergistically toward a greater renal injury than the sum of their independent effects. Finally, a decrease in adenosine production [43] and the homocysteinylation of some plasma proteins [44-45] during HHcy may also activate or promote the sclerotic process in the glomeruli. Through these mechanisms, elevated homocysteine may promote acceleration of intrarenal arteriosclerotic vascular lesions, resulting in the development of renal function deterioration.

Plasma Homocysteine with Renal

Function Aggravation
As homocysteine is excreted mainly by the kidney, impaired renal excretion was previously thought as a significant determinate of tHcy levels in plasma. Recently, there is growing evidence that the potential causes of HHcy in renal failure are decreased renal or extrarenal metabolism as a result of uremic toxicity, while homocysteine excretion is negligible [46]. In this large population-based study, the association between elevated tHcy levels and rapid eGFR decline or higher CKD development was observed independently of baseline renal function, reflecting the fact that these associations are not simply the consequence of reduced renal function. This result is consistent with the hypothesis that homocysteine is an important pathogenic factor for glomerular damage. Therefore, it is reasonable to conclude that HHcy and reduced renal function may interact with each other to form a vicious circle toward consequent glomerulosclerosis and CKD. Our study also found that old age, blood glucose, and central pulse pressure were associated with renal function change besides tHcy, but in this cohort, tHcy levels remained independent prediction of research end points in the final models after additional adjustments for these confounding factors.

Given that plasma tHcy may contribute to renal function deterioration, it may be supposed that lowering the levels of homocysteine may reduce the risk of renal function decline and incident CKD. Yet, the available intervention studies regarding the effect of homocysteinelowering therapies on CKD progression are controversial $[20,29,47]$. The CSPPT study which was conducted in 15,104 Chinese adults with hypertension indicated that folic acid supplementation could significantly delay the progression of CKD among patients with mild-to-moderate CKD [29]. However, in the HOST study on 2,056 participants with advanced CKD or end-stage renal disease, treatment with high doses of folic acid and B vitamins failed to delay the time to require maintenance dialysis [20]. The DIVINe study investigated 238 patients with diabetic nephropathy and unexpectedly showed that high doses of B vitamins compared with placebo resulted in a greater decrease in GFR and an increase in vascular events [47]. However, in animal experiments [48-49], daily supplementation with folic acid significantly lowered plasma tHcy concentrations and prevented the functional and histological markers of HHcy-induced nephropathy. The nonhomogeneous population selection and different vitamin B doses among studies may result in such conflicting results. Further intervention research studies are needed to demonstrate whether homocysteine-lowering therapies could reduce the incidence or delay the progression of CKD. 
Our study also has several limitations. First, urinary albumin excretion rate, an important marker of kidney damage, was not measured during follow-up. This may result in underestimating the prevalence of CKD. Second, we did not perform detection using cystatin $\mathrm{C}$, a potentially more accurate measure of kidney function in the elderly than serum creatinine. Third, we had no data on vitamin status, including the status of folic acid, vitamin $\mathrm{B}$, or MTHFR polymorphism, which may affect tHcy levels during the observation period. However, regarding the relationship between vitamin supplementation and CKD progression, available interventional studies have demonstrated no clear benefit or even harmful effects on renal outcomes [50]. Finally, the study population was restricted to Chinese community-based residents of Han origin, and its generalizability to other demographic groups should be done with caution.

\section{Conclusion}

In a prospective cohort of community-dwelling population, we found that elevated plasma homocysteine levels were an independent risk factor for accelerated renal function decline and future incidence of CKD. This study adds credence to the evidence from previous studies that tHcy could be a possible biomarker for renal disease progression. Further investigations are needed to explore which homocysteine-lowering treatments will improve long-term renal outcomes.

\section{Acknowledgement}

We thank our colleagues at the Department of Laboratory Medicine of the PLA General Hospital for their assistance with biochemical measurements.

\section{Statement of Ethics}

The study was approved by the Ethics Committee of the Chinese PLA General Hospital (S2016-056-02), and all participants gave written informed consent at time of enrollment.

\section{Conflict of Interest Statement}

The authors declare that they have no conflicts of interest.

\section{Funding Sources}

This study was funded by the National Key R\&D Program of China, No. 2018YFC2002102 and No. 2020YFC2004805.

\section{Author Contributions}

Study design: P.Y. and W.K.X.; data analysis: W.K.X., F.W., and Y.Y.B.; manuscript writing: W.K.X. Data collection supervision: R.H.C. and X.N.W. All authors approved the final version of the manuscript.

\section{References}

1 Long Y, Nie J. Homocysteine in renal injury. Kidney Dis. 2016 Jun;2(2):80-7.

2 Okabayashi Y, Tsuboi N, Kanzaki G, Sasaki T, Haruhara K, Koike K, et al. Aging versus hypertension: an autopsy study of sclerotic renal histopathological lesions in adults with normal renal function. Am J Hypertens. 2019 Jun;32(7):676-83.

3 de Boer IH, Rue TC, Hall YN, Heagerty PJ, Weiss NS, Himmelfarb J. Temporal trends in the prevalence of diabetic kidney disease in the United States. JAMA. 2011 Jun;305(24): 2532-9.

4 Mora-Gutiérrez JM, Slon Roblero MF, Castaño Bilbao I, Bautista DI, Coloma JA, Velilla NM. (Chronic kidney disease in the elderly patient). Rev Esp Geriatr Gerontol. 2017 May-Jun;52(3):152-8.

5 Welch GN, Loscalzo J. Homocysteine and atherothrombosis. N Engl J Med. 1998 Apr; 338(15):1042-50.
6 Homocysteine Studies Collaboration. Homocysteine and risk of ischemic heart disease and stroke: a meta-analysis. JAMA. 2002 Oct; 288(16):2015-22

7 Wang C, Han L, Wu Q, Zhuo R, Liu K, Zhao $\mathrm{J}$, et al. Association between homocysteine and incidence of ischemic stroke in subjects with essential hypertension: a matched casecontrol study. Clin Exp Hypertens. 2015; 37(7):557-62.

$8 \mathrm{Fu} \mathrm{HJ}$, Zhao LB, Xue JJ, Wu ZX, Huang YP, Liu W, et al. Elevated serum homocysteine (Hcy) levels may contribute to the pathogenesis of cerebral infarction. J Mol Neurosci. $2015 \mathrm{Jul} ; 56(3): 553-61$.

9 Ye ZC, Zhang QZ, Li Y, Wang C, Zhang J, Ma XX, et al. High prevalence of hyperhomocysteinemia and its association with target organ damage in Chinese patients with chronic kidney disease. Nutrients. 2016 Oct; 8(10):645.
10 Lai SH, Tsai YW, Chen YC, Chang SS. Obesity, hyperhomocysteinaemia and risk of chronic kidney disease: a population-based study. Fam Pract. 2018 May;35(3):259-65.

11 Cohen E, Margalit I, Shochat T, Goldberg E, Krause I. The relationship between the concentration of plasma homocysteine and chronic kidney disease: a cross sectional study of a large cohort. J Nephrol. 2019 Oct;32(5): 783-9.

12 Pushpakumar S, Kundu S, Sen U. Hydrogen sulfide protects hyperhomocysteinemia-induced renal damage by modulation of caveolin and eNOS interaction. Sci Rep. 2019 Feb; 9(1):2223.

13 Yi F, Li PL. Mechanisms of homocysteine-induced glomerular injury and sclerosis. Am J Nephrol. 2008;28(2):254-64. 
14 Amos L, Cohen E, Levi M, Goldberg E, Garty $\mathrm{M}$, Krause I. Elevated serum homocysteine is a predictor of accelerated decline in renal function and chronic kidney disease: a historical prospective study. Eur J Intern Med. 2014 Dec;25(10):951-5.

15 Sarnak MJ, Wang SR, Beck GJ, Kusek JW, Selhub J, Greene T, et al. Homocysteine, cysteine, and $\mathrm{B}$ vitamins as predictors of kidney disease progression. Am J Kidney Dis. 2002 Nov;40(5):932-9.

16 Hao L, Ma J, Zhu J, Stampfer MJ, Tian Y, Willett WC, et al. High prevalence of hyperhomocysteinemia in chinese adults is associated with low folate, vitamin B-12, and vitamin B-6 status. J Nutr. 2007 Feb;137(2):407-13.

17 Xiao W, Wen Y, Ye P, Wang F, Cao R, Bai Y, et al. Noninvasive central pulse pressure is an independent determinant of renal function. J Clin Hypertens. 2020 Feb;22(2):234-42.

18 Araki A, Sako Y. Determination of free and total homocysteine in human plasma by highperformance liquid chromatography with fluorescence detection. J Chromatogr. 1987 Nov; 422:43-52.

19 Marti F, Vollenweider P, Marques-Vidal PM, Mooser V, Waeber G, Paccaud F, et al. Hyperhomocysteinemia is independently associated with albuminuria in the population-based CoLaus study. BMC Public Health. 2011 Sep; 11:733.

20 Jamison RL, Hartigan P, Kaufman JS, Goldfarb DS, Warren SR, Guarino PD, et al. Effect of homocysteine lowering on mortality and vascular disease in advanced chronic kidney disease and end-stage renal disease: a randomized controlled trial. JAMA. 2007 Sep; 298(10): 1163-70

21 Levey AS, Stevens LA, Schmid CH, Zhang YL, Castro AF 3rd, Feldman HI, et al. A new equation to estimate glomerular filtration rate. Ann Intern Med. 2009 May;150(9):604-12.

22 Kuczera P, Kwiecień K, Adamczak M, Bączkowska T, Gozdowska J, Madziarska K, et al. Different relevance of peripheral, central or nighttime blood pressure measurements in the prediction of chronic kidney disease progression in patients with mild or no-proteinuria. Kidney Blood Press Res. 2018;43(3):735-43.

23 Peralta CA, Katz R, Sarnak MJ, Ix J, Fried LF, Boer ID, et al. Cystatin C identifies chronic kidney disease patients at higher risk for complications. J Am Soc Nephrol. 2011 Jan;22(1): 147-55.

24 Weekley CC, Peralta CA. Advances in the use of multimarker panels for renal risk stratification. Curr Opin Nephrol Hypertens. 2012 May;21(3):301-8

25 Fox CS, Gona P, Larson MG, Selhub J, Tofler G, Hwang SJ, et al. A multi-marker approach to predict incident CKD and microalbuminuria. J Am Soc Nephrol. 2010 Dec;21(12): 2143-9.
26 Gao X, Yao M, McCrory MA, Ma GS, Li YP, Roberts SB, et al. Dietary pattern is associated with homocysteine and B vitamin status in an urban Chinese population. J Nutr. 2003 Nov; 133(11):3636-42

27 Li ZH, Sun L, Zhang HY, Liao YH, Wang DW, Zhao BR, et al. Elevated plasma homocysteine was associated with hemorrhagic and ischemic stroke, but methylenetetrahydrofolate reductase gene $\mathrm{C} 677 \mathrm{~T}$ polymorphism was a risk factor for thrombotic stroke: a multicenter case-control study in China. Stroke. 2003 Sep;34(9):2085-90.

28 Botto LD, Yang Q. 5,10-methylenetetrahydrofolate reductase gene variants and congenital anomalies: a huge review. Am J Epidemiol. 2000 May;151(9):862-77.

29 Xu X, Qin X, Li Y, Sun D, Wang J, Liang M, et al. Efficacy of folic acid therapy on the progression of chronic kidney disease: the renal substudy of the China stroke primary prevention trial. JAMA Intern Med. 2016 Oct; 176(10):1443-50.

30 Ninomiya T, Kiyohara Y, Kubo M, Tanizaki Y, Tanaka K, Okubo K, et al. Hyperhomocysteinemia and the development of chronic kidney disease in a general population: the hisayama study. Am J Kidney Dis. 2004 Sep; 44(3):437-45

31 Xie D, Yuan Y, Guo J, Yang S, Xu X, Wang Q, et al. Hyperhomocysteinemia predicts renal function decline: a prospective study in hypertensive adults. Sci Rep. 2015 Nov;5:16268.

32 Samuelsson O, Lee DM, Attman PO, Gibson CK, Mullen JK, Larsson R, et al. The plasma levels of homocysteine are elevated in moderate renal insufficiency but do not predict the rate of progression. Nephron. 1999;82(4): 306-11.

33 van Dijk SC, Enneman AW, Swart KM, van Wijngaarden JP, Ham AC, de Jonge R, et al. Effect of vitamin B12 and folic acid supplementation on biomarkers of endothelial function and inflammation among elderly individuals with hyperhomocysteinemia. Vasc Med. 2016 Apr;21(2):91-8.

34 de Andrade CR, Leite PF, Montezano AC, Casolari DA, Yogi A, Tostes RC, et al. Increased endothelin-1 reactivity and endothelial dysfunction in carotid arteries from rats with hyperhomocysteinemia. Br J Pharmacol. 2009 Jun;157(4):568-80.

35 Yang Q, He G-W. Imbalance of homocysteine and H2S: significance, mechanisms, and therapeutic promise in vascular injury. Oxid Med Cell Longev. 2019 Nov:7629673.

36 Perna AF, Luciano MG, Ingrosso D, Pulzella $\mathrm{P}$, Sepe I, Lanza D, et al. Hydrogen sulphidegenerating pathways in haemodialysis patients: a study on relevant metabolites and transcriptional regulation of genes encoding for key enzymes. Nephrol Dial Transplant. 2009 Dec;24(12):3756-63.
37 Sen U, Munjal C, Qipshidze N, Abe O, Gargoum R, Tyagi SC. Hydrogen sulfide regulates homocysteine-mediated glomerulosclerosis. Am J Nephrol. 2010;31(5):442-55.

38 Bidani AK, Polichnowski AJ, Loutzenhiser R, Griffin KA. Renal microvascular dysfunction, hypertension and CKD progression. Curr Opin Nephrol Hypertens. 2013 Jan;22(1):1-9.

39 Ostrakhovitch EA, Tabibzadeh S. Homocysteine in chronic kidney disease. Adv Clin Chem. 2015;72:77-106.

40 Emsley AM, Jeremy JY, Gomes GN, Angelini $\mathrm{D}$, Plane F. Investigation of the inhibitory effects of homocysteine and copper on nitric oxide-mediated relaxation of rat isolated aorta. Br J Pharmacol. 1999 Feb;126(4):1034-40.

41 Zhong MF, Zhao YH, Xu H, Tan X, Wang YK, Wang WZ. The cardiovascular effect of systemic homocysteine is associated with oxidative stress in the rostral ventrolateral medulla. Neural Plast. 2017;2017:3256325.

42 Shi WR, Zhou YP, Wang HY, Sun YX, Chen YH. Synergistic interaction of hypertension and hyperhomocysteinemia on chronic kidney disease: findings from the National health and nutrition examination survey 1999-2006. J Clin Hypertens. 2019 Oct;21(10):1567-77.

43 Chen YF, Li PL, Zou AP. Effect of hyperhomocysteinemia on plasma or tissue adenosine levels and renal function. Circulation. 2002 Sep;106(10):1275-81.

44 Perna AF, Satta E, Acanfora F, Lombardi C, Ingrosso D, De Santo NG. Increased plasma protein homocysteinylation in hemodialysis patients. Kidney Int. 2006 Mar;69(5):869-76.

45 Fu Y, Wang X, Kong W. Hyperhomocysteinaemia and vascular injury: advances in mechanisms and drug targets. Br J Pharmacol. 2018 Apr;175(8):1173-89.

46 Perna AF, Ingrosso D, Satta E, Lombardi C, Acanfora F, De Santo NG. Homocysteine metabolism in renal failure. Curr Opin Clin Nutr Metab Care. 2004 Jan;7(1):53-7.

47 House AA, Eliasziw M, Cattran DC, Churchill DN, Oliver MJ, Fine A. Effect of B-vitamin therapy on progression of diabetic nephropathy: a randomized controlled trial. JAMA. 2010 Apr;303(16):1603-9.

48 Cao L, Lou X, Zou Z, Mou N, Wu W, Huang $\mathrm{X}$, et al. Folic acid attenuates hyperhomocysteinemia-induced glomerular damage in rats. Microvasc Res. 2013 Sep;89:146-52.

49 Gao N, Zhang Y, Lei L, Li L, Cao P, Zhao X, et al. Low doses of folic acid can reduce hyperhomocysteinemia-induced glomerular injury in spontaneously hypertensive rats. Hypertens Res. 2020 Nov;43(11):1182-91.

50 Capelli I, Cianciolo G, Gasperoni L, Zappulo F, Tondolo F, Cappuccilli M, et al. Folic acid and vitamin $\mathrm{B} 12$ administration in $\mathrm{CKD}$, why not? Nutrients. 2019 Feb;11(2):383.
Plasma Homocysteine with Renal Function Aggravation
Kidney Blood Press Res 2021;46:541-549 DOI: $10.1159 / 000514360$ 